\title{
Sonographic Diagnosis of Preduodenal Portal Vein in Children
}

\author{
Vassil Zefov, ${ }^{1 *}$ Fatma Abdelrady, ${ }^{1}$ Mamoun $\mathrm{AM},{ }^{2}$ Diary $\mathrm{AR}^{2}$ \\ ${ }^{1}$ Pediatric Radiology, Latifa Women \& Children Hospital, Dubai \\ ${ }^{2}$ Pediatric surgery, Latifa Women \& Children Hospital, Dubai
}

\begin{abstract}
Purpose: To clarify the role of sonography in diagnosis of Preduodenal Portal Vein (PDPV) in children.

Material and methods: We present two cases with PDPV in children proved with surgery. The first case was diagnosed retrospectively, the second one prospectively.
\end{abstract}

Results: In both cases the PDPV appears above the duodenum with convexity which is opposite of normal concave configuration of PV. Both cases appear with multiple congenital association anomalies.

Conclusions: The sonography is safe, convenient, fast, time- effective and cost- effective method for precise diagnosis of PDPV in children.

Keywords: Portal vein, Preduodenal portal vein. Annular pancreas, Duodenal web

Abbreviations: PV, portal vein; PDPV, preduodenal portal vein; AP, annular pancreas; DW, duodenal web

\section{Introduction}

Preduodenal portal vein (PDPV) is a rare anomaly in which the portal vein passes anterior to the duodenum rather than posteriorly. Generally asymptomatic, PDPV may rarely cause duodenal obstruction or may coexist with other anomalies. The PDPV can exist with Malrotation, ${ }^{1}$ Duodenal web, ${ }^{1,2}$ Situs Inversus, ${ }^{4}$ Volvulus, ${ }^{5}$ Polisplenia, ${ }^{6,7}$ Annular pancreas and Callosal agenesis ${ }^{8}$ Most of the authors stress point the significance of preoperative information of PDPV existence for the surgeons rather than as a main reason for duodenal obstruction. The imaging arsenal for diagnosis of PDPV includes venography, sonography, contrast meal with follow through, CT and MRI.

\section{Case 1}

27 days old female with multiple congenital anomalies, transferred to LWCH for evaluation and treatment. The admission radio graph of abdomen shows features of Cauda regression syndrome with absent limbo sacral segment of the spine, fused iliac bones, separated ischial bones, fracture of left femur, gas distended stomach and duodenum suggestive of partial duodenal obstruction. Bowels are seen herniated through the pelvis out (Figure 1). The first abdominal sonography shows distended duodenum with beak toward the pancreatic head, surrounded with pancreatic tissue Figure 2 suggestive of the reason for partial duodenal obstruction. The renal sonography shows fused kidneys at midline seen better posteriorly due to absent lumbar spine (Figure 3). The next step was Contrast meal with follow through: The stomach and duodenum appear dilated and separated by deep incisura which brings in mind possibility of vascular impression. In addition, the duodenum shows beak (Figure 4).

\begin{tabular}{|l|l|}
\hline Quick Response Code: & $\begin{array}{l}\text { *Corresponding author: Vassil Zefov, Pediatric Radiology, DHA, Latifa Women \& Children } \\
\text { Hospital, Dubai } \\
\text { Received: } 23 \text { November, } 2020 \\
\text { Citation: Vassil Z, Fatma A, Mamoun AM, Diary AR. Sonographic Diagnosis of Preduode- } \\
\text { nal Portal Vein in Children. SOJ Pedia Clin Neonato. 2020;1(1):1-5. DOI: 10.53902/SO- } \\
\text { JCN.2020.01.000505 }\end{array}$ \\
\hline
\end{tabular}



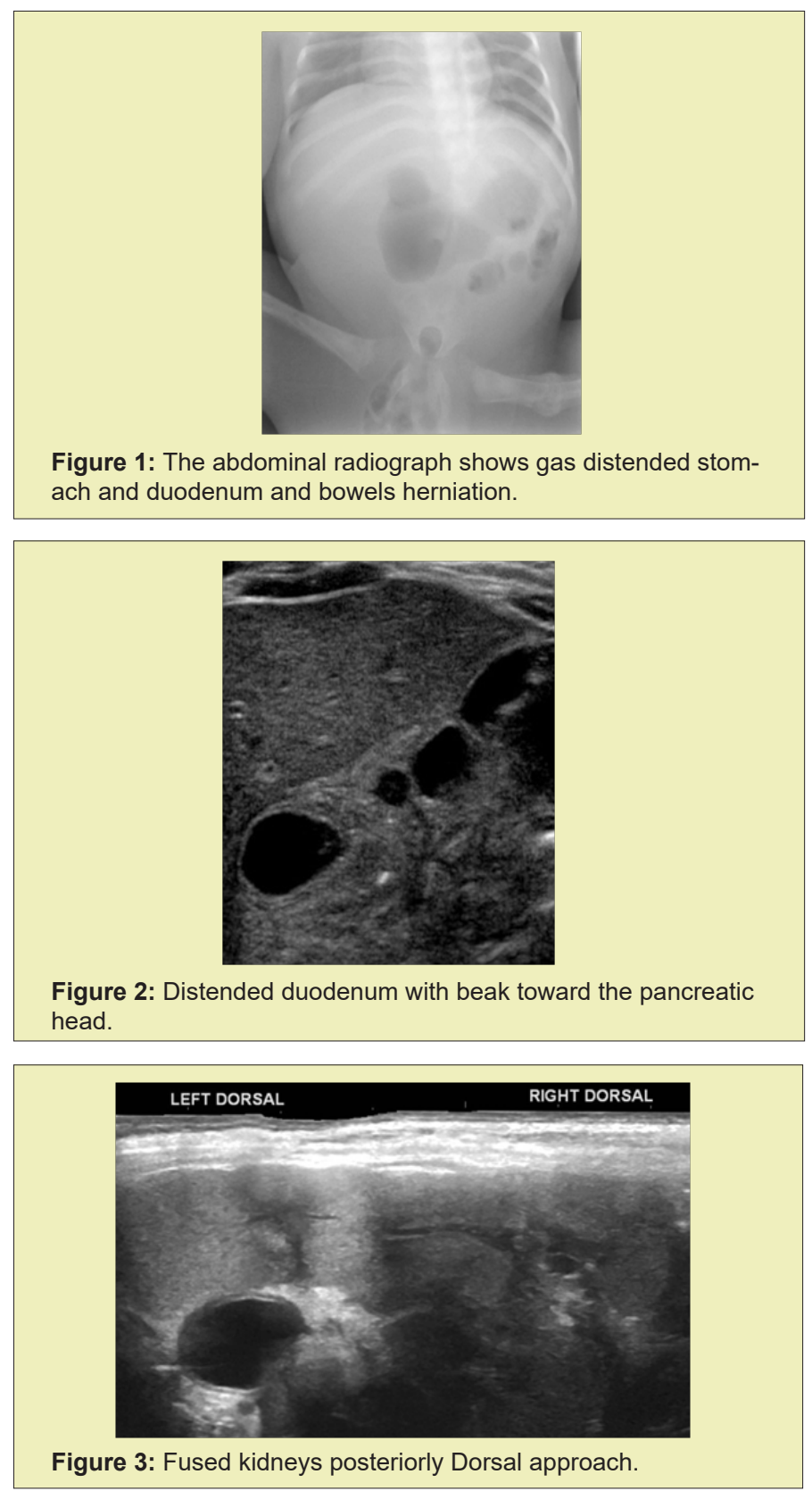

Figure 3: Fused kidneys posteriorly Dorsal approach.

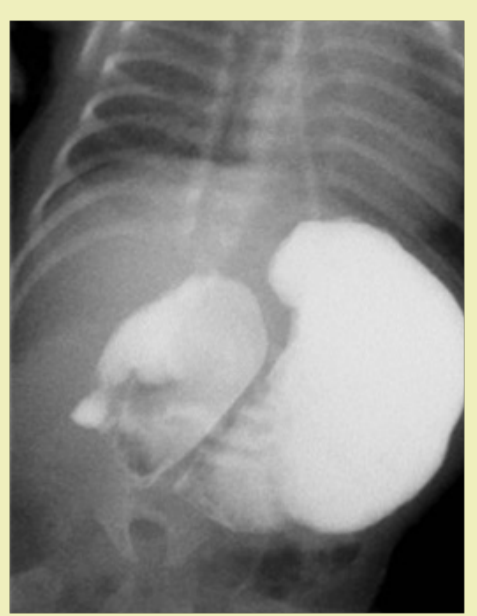

Figure 4: Deep incisura separates the Stomach and duodenum.
The follow through shows opacified, herniated bowels trough the pelvis (Figure 5).

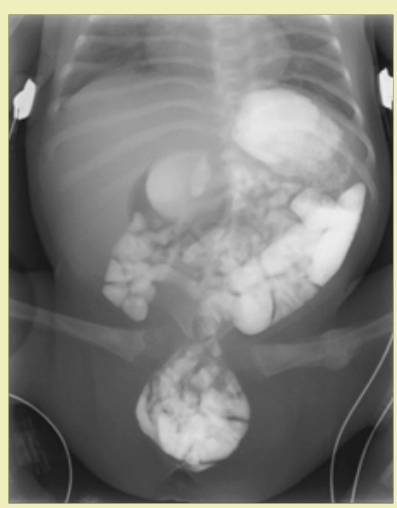

Figure 5: The follow through shows herniated bowels through the pelvis.

The findings prompted us to revise the previous abdominal sonography and surprisingly we discover retrospectively missed sign of arching portal vein above the duodenum which is obvious abnormal (Figure 6). As the duodenum appears distended distally from portal vein it was assumed the portal vein is not obstructing the duodenum. The possibility of AP or DW arise, and the patient was sent for surgical treatment without any additional exams. The surgical report reveals: Partial duodenal obstruction, AP and PDPV. Performed surgery- Duodeno-Jejunostomy (Diamond anastomosis)

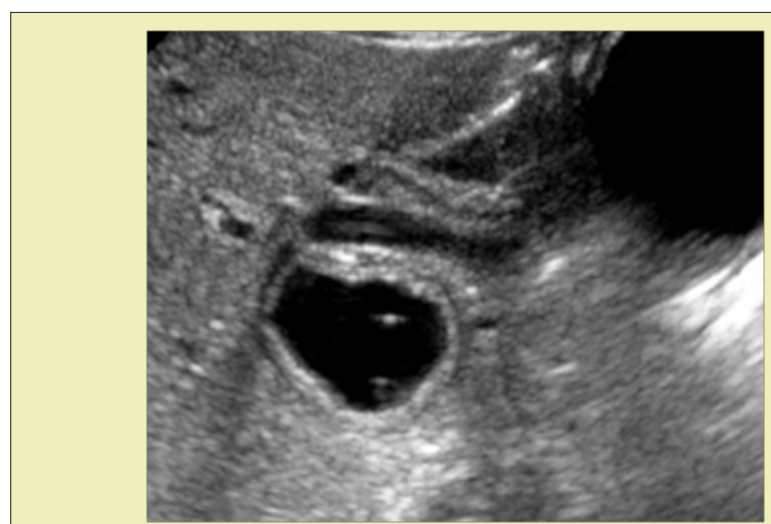

Figure 6: The Portal vein appears arching above the distended duodenum $=$ PDPV.

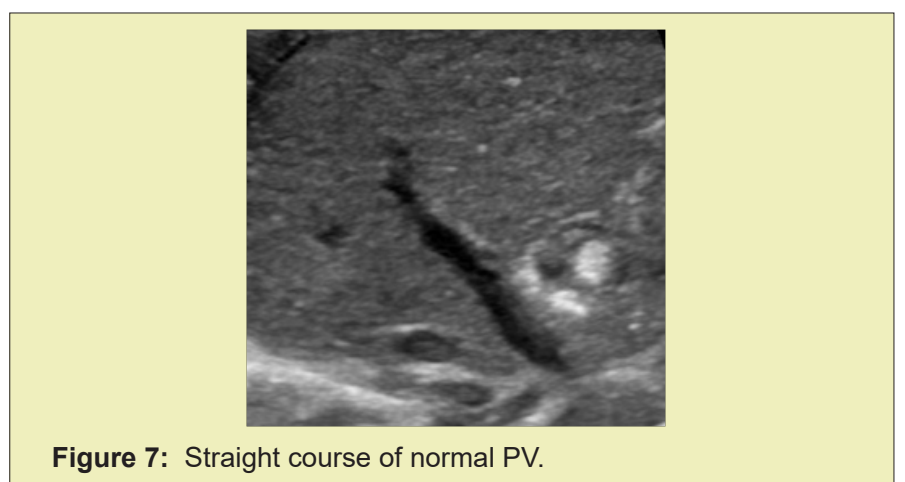

The result of our firs case of PDPV prompted us to do prospective study regarding the normal relationship between the extrahepatic portal vein and duodenum. We examine Sonographically 100 
random cases between 1 day and 13years - 50 boys and 50 girls. The normal PV appears as strait (Figure 7). Or concave configuration (Figures 8\&9) depending on the scanning. The duodenum is always above the PV.
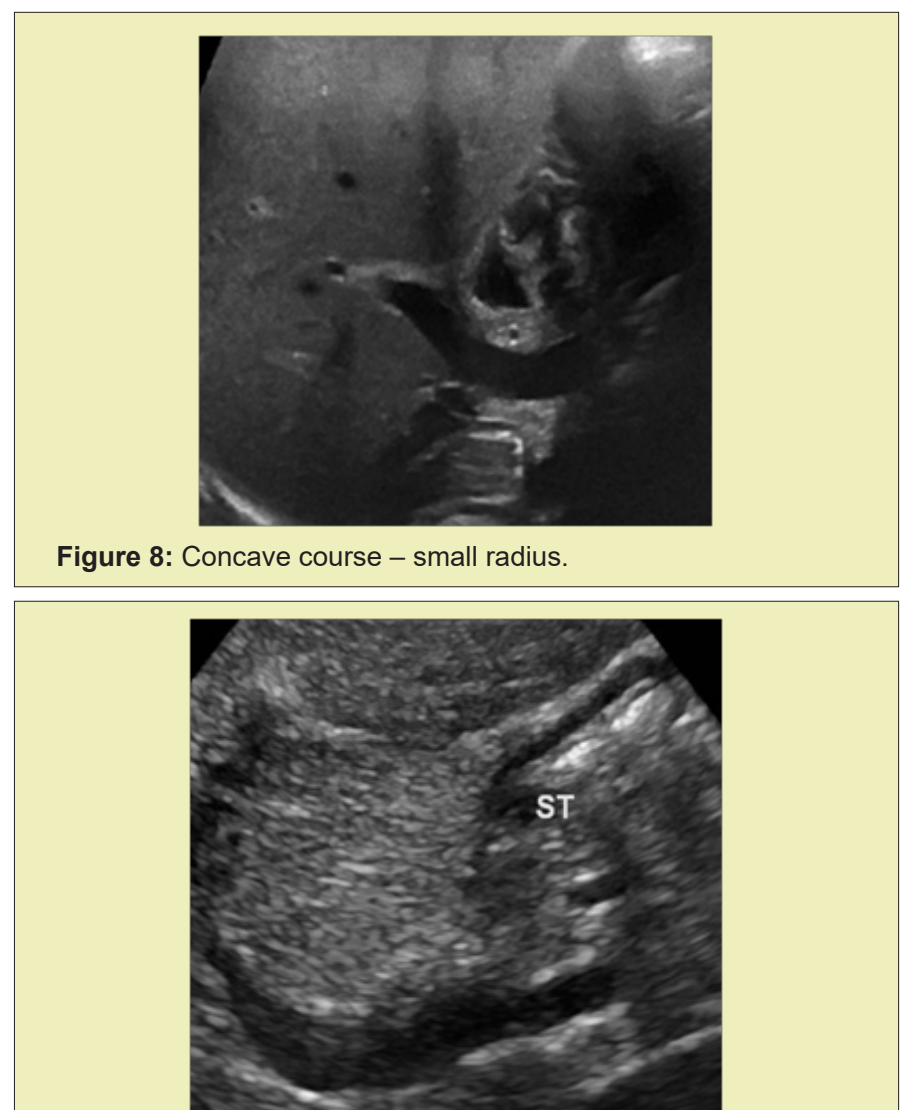

Figure 9: Concave course-bigger radius.

\section{Case 2}

Our second case was depicted antenatally as double bubble sign suggestive of duodenal atresia (Figure 10).

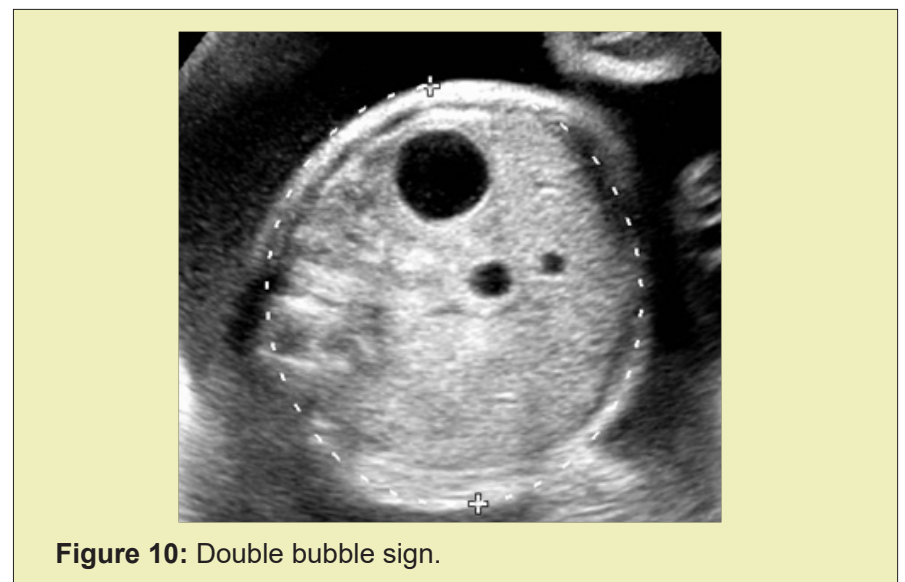

The postnatal abdominal radiograph shows gas distended stomach and duodenum suggestive of Duodenal obstruction (Figure 11). The preoperative protocol requires examinations of the brain and abdomen. The brain sonography shows Agenesis of corpus callosum with US Figures $12 \& 13$ proved with MRI Figures 1419.
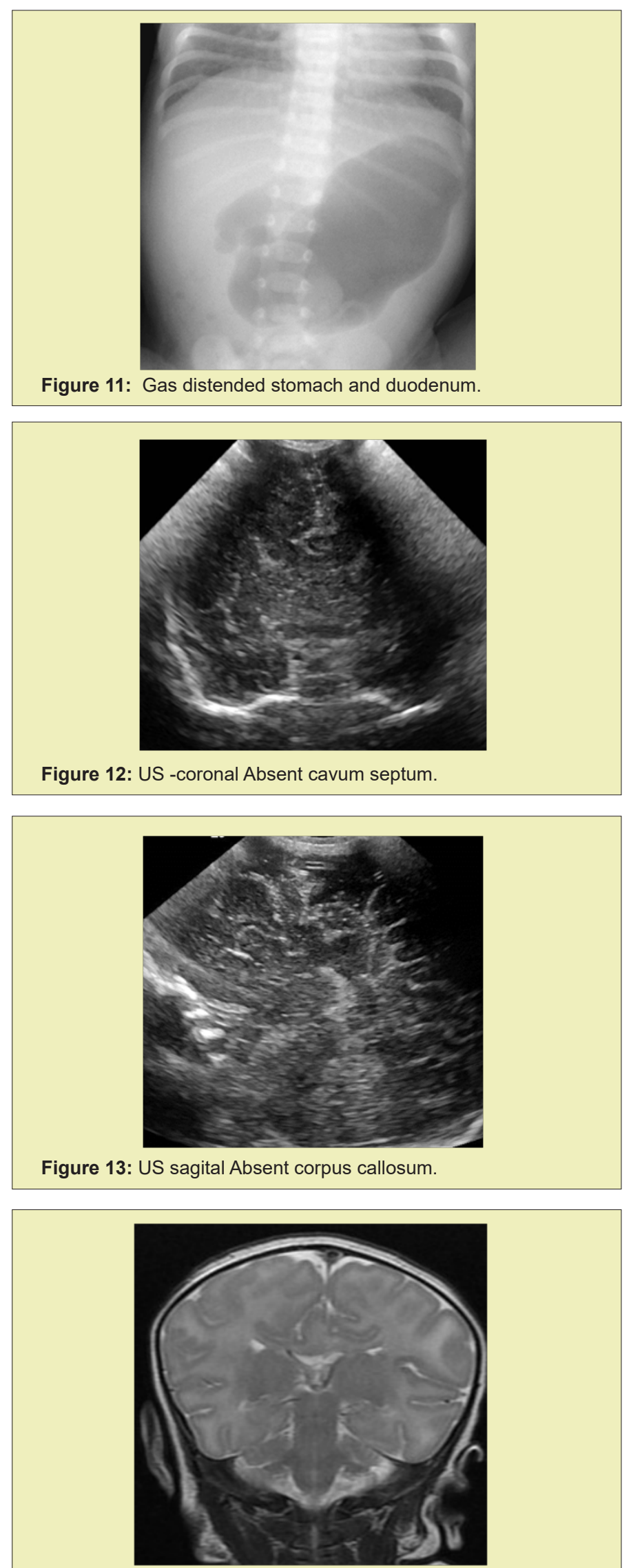

Figure 14: MR coronal - widely separated Anterior horns. 


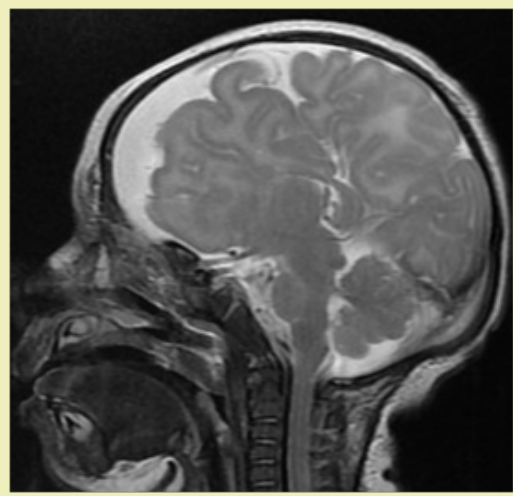

Figure 15: MR sagital -absent corpus callosum.
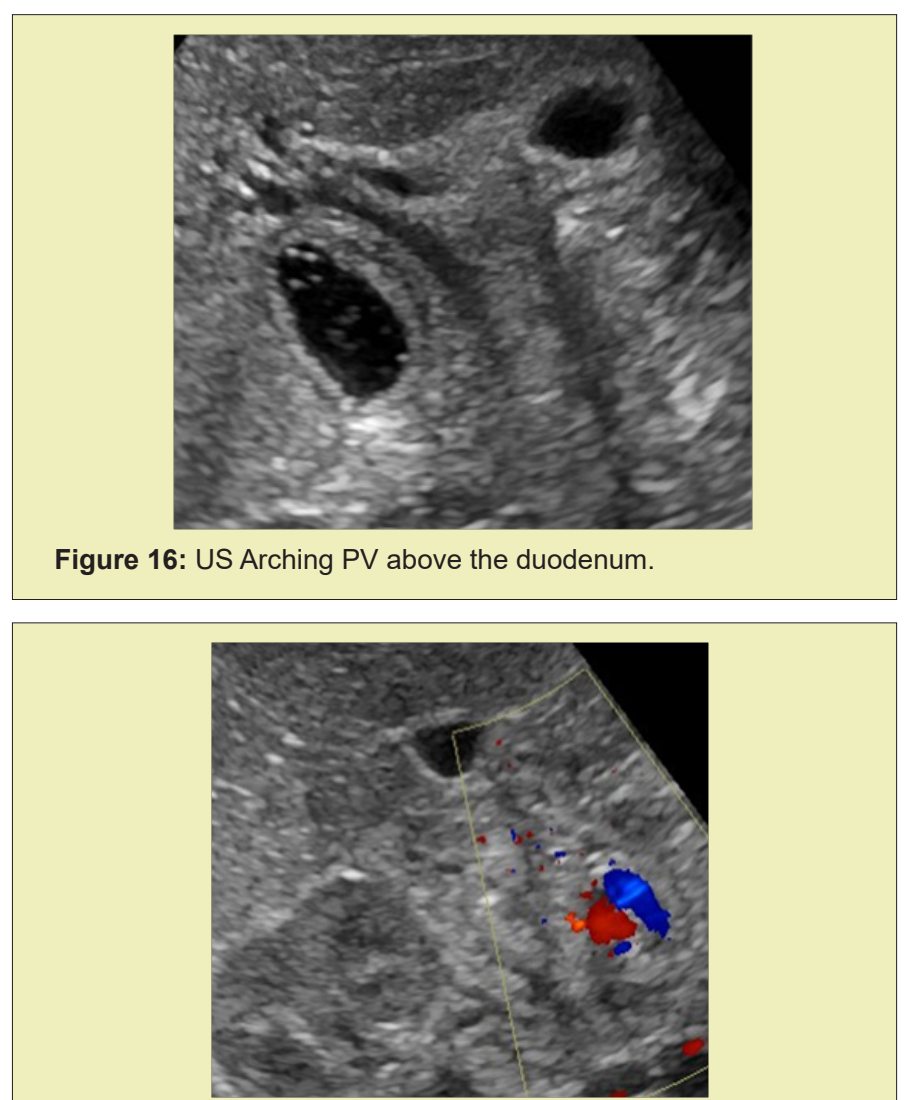

Figure 17: Mesenteric vein - on the left side of mesenteric artery.

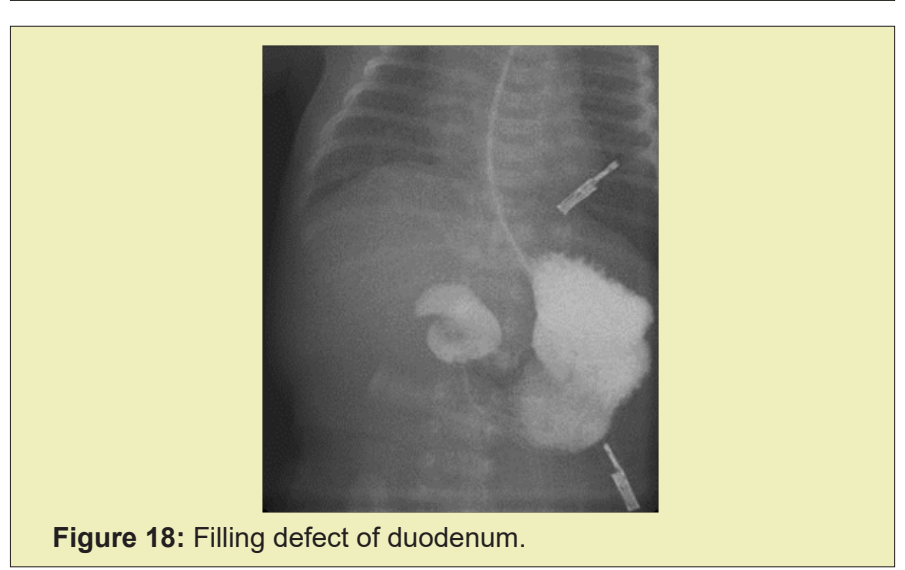

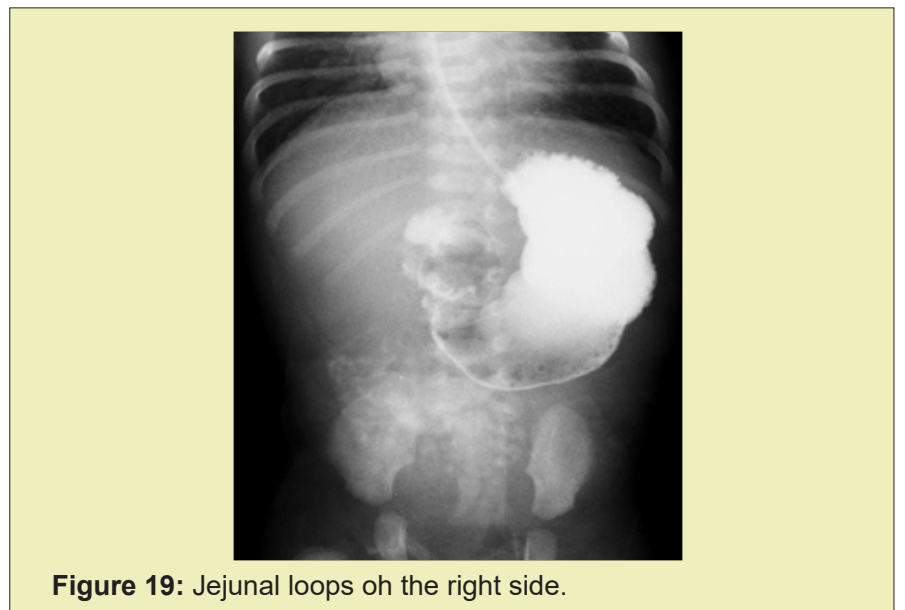

The abdominal sonography shows typical arching of PV above the duodenum suggestive of PDPV. In addition, the mesenteric vein appears on the left side of mesenteric artery giving image of whirlpool suggestive of Malrotation. The follow contrast meal and follow through show contrasted jejunal loops on the right side suggestive of Malrotation and duodenum appears with filling defect suggestive of DW. The patient was sent for surgical treatment. The surgical report came surprising revealing: Malrotation (Figure 20). PDPV , AP and DW Figures 21\&22, The DW was located centrally in the duodenal lumen, performed surgery - Duodeno -duodenostomy over the DW - as PDPV appears nonobstructive.

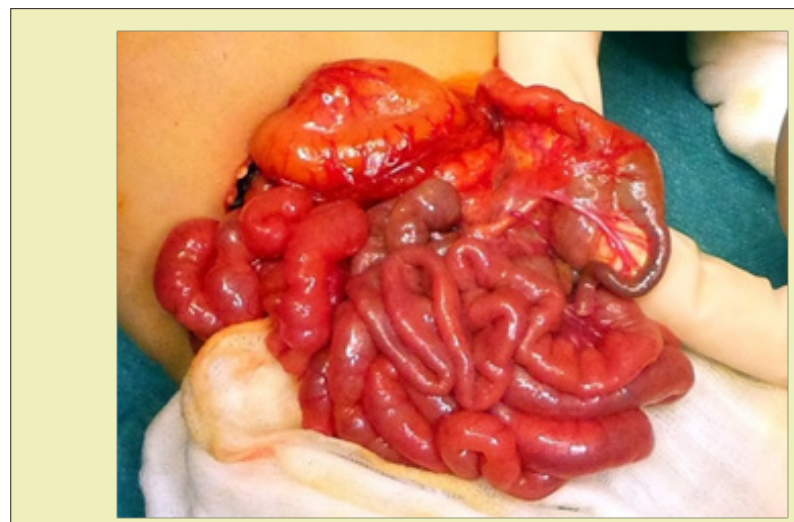

Figure 20: Appendix on the left side- Malrotation.

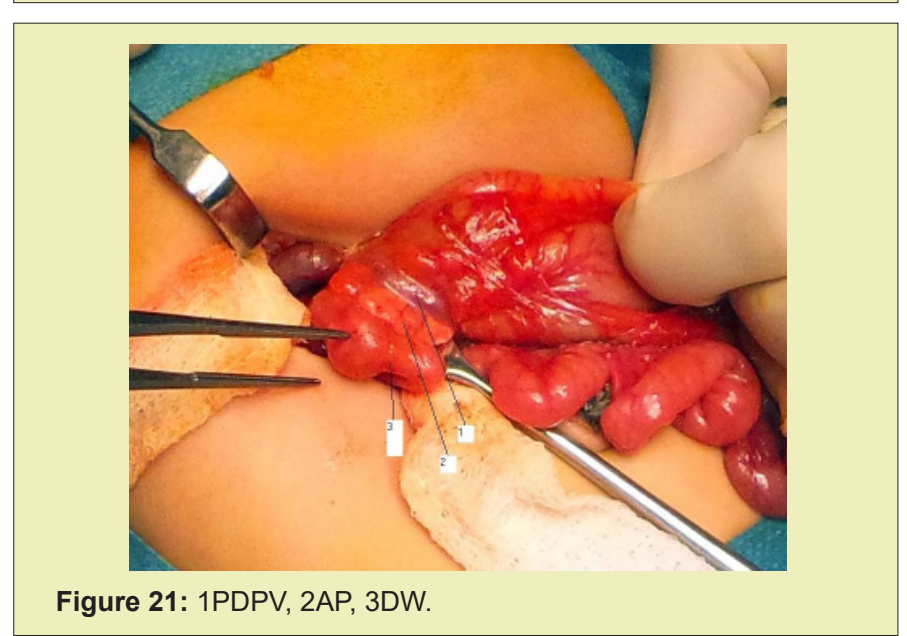




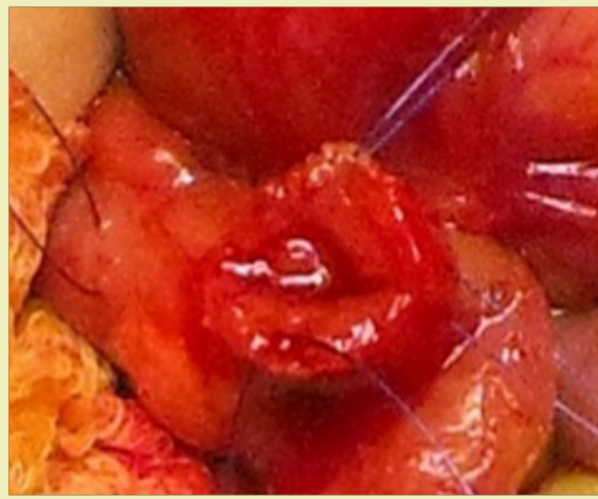

Figure 22: Arrow - DW in the lumen of duodenum.

\section{Discussion}

Both Cases show multiple congenital abnormalities.

The case 1 appears associated with 5 congenital abnormalities: Cauda regression syndrome, Fused iliac bones,

Fused kidneys, AP and herniated bowels.

The case 2 appears associated with 4 congenital anomalies: AP, Callosal agenesis, Malrotation and DW.

If adding PDPV to both cases the terminology is complicated with Hexi and Penta.

\section{Conclusions}

The sonographic diagnosis of PDPV based on the arching PV above the duodenum is convenient, safe, time -effective and costeffective method.

Giving important information to the surgeons is great help for the preoperative planning.
It complies completely with "Image Gently" principles.

If we discover the anomaly of PDPV we should try to check for another anomaly.

\section{Acknowledgments}

None.

\section{Funding}

None.

\section{Conflicts of interest}

The authors report no financial interests or potential conflicts of interest.

\section{References}

1. Singal AK, Ramu C, Paul S, et al. Preduodenal portal vein in association with midgut malrotation and duodenal web- triple anomaly? J Pediatr Surg. 2009;44:E5-E7.

2. Golombek S, Bilgi J, Ukabiala O. Duodenal web with preduodenal portal vein. Iowa Med. 1995;85:247-9.

3. Choi SO, Park WH. Preduodenal portal vein: a cause of prenatally diagnosed duodenal obstruction. J Pediatr Surg. 1995;30:1521-2.

4. Ram Mohan Shukla, Partha Pratik Mukherjee, B Mukhopadhyay, et al. Congenital Duodenal Obstruction with Preduodenal Portal Vein and Situs Inversus Totalis: Report of Two Cases and Literature Review. Indian J Surg. 2013;75(Suppl 1):S74-S76.

5. Toshihiko Watanabe, Miwako Nakano, Kazuki Yamazawa, et al. Neonatal Intestinal Volvulus and Preduodenal Portal Vein Associated with Situs Ambiguous: Report of a Case. Surg Today. 2011;41:726-729.

6. P De Wailly, P Metzler, N Sautot-Vial, Pre-duodenal portal vein in polysplenia syndrome: clinical effects and surgical application. Surg Radiol Anat. 2011;33(5):451-454.

7. McCarten KM, Teele RL. Preduodenal portal vein: Venography, ultrasonography, and review of the literature. Ann Radio1.1978;21:155160.

8. Zefov, Samara, Nasreen AM, et al. Sonographic diagnosis of Preduodenal portal vein in children. PP Presentation at AOSPR, Borneo. 2016. 